\title{
Differential Attacks against Stream Cipher ZUC*
}

\author{
Hongjun Wu, Tao Huang, Phuong Ha Nguyen, Huaxiong Wang, and San Ling \\ Division of Mathematical Sciences, \\ School of Physical and Mathematical Sciences \\ Nanyang Technological University, Singapore \\ $\{$ wuhj, huangtao, ng007ha, hxwang, lingsan $\}$ @ntu .edu.sg
}

\begin{abstract}
Stream cipher ZUC is the core component in the $3 \mathrm{GPP}$ confidentiality and integrity algorithms 128-EEA3 and 128-EIA3. In this paper, we present the details of our differential attacks against ZUC 1.4. The vulnerability in ZUC 1.4 is due to the non-injective property in the initialization, which results in the difference in the initialization vector being cancelled. In the first attack, difference is injected into the first byte of the initialization vector, and one out of $2^{15.4}$ random keys result in two identical keystreams after testing $2^{13.3}$ IV pairs for each key. The identical keystreams pose a serious threat to the use of ZUC 1.4 in applications since it is similar to reusing a key in one-time pad. Once identical keystreams are detected, the key can be recovered with average complexity $2^{99.4}$. In the second attack, difference is injected into the second byte of the initialization vector, and every key can result in two identical keystreams with about $2^{54}$ IVs. Once identical keystreams are detected, the key can be recovered with complexity $2^{67}$. We have presented a method to fix the flaw by updating the LFSR in an injective way in the initialization. Our suggested method is used in the later versions of ZUC. The latest ZUC 1.6 is secure against our attacks.
\end{abstract}

\section{Introduction}

Comparing to block ciphers, dedicated stream ciphers normally require less computation for achieving the same security level. Stream ciphers are widely used in applications. For example, RC4 [10] is used in SSL and WEP, and A5/1 [8] is used in GSM (the Global System for Mobile Communications). But the use of RC4 in WEP is insecure [7], and A5/1 is very weak [4]. ECRYPT (2004-2008) has organised the eSTREAM competition, which stimulated the study on stream ciphers, and a number of new stream ciphers were proposed [1 3, 5, 6, 9, 15].

The 3rd Generation Partnership Project (3GPP) was set up for making globally applicable $3 \mathrm{G}$ mobile phone system specifications based on the GSM specifications. Stream cipher ZUC was designed by the Data Assurance and Communication Security Research Center of the Chinese Academy of Sciences.

\footnotetext{
* This research is supported by the National Research Foundation Singapore under its Competitive Research Programme (CRP Award No. NRF-CRP2-2007-03) and Nanyang Technological University NAP startup grant (M4080529.110).
}

X. Wang and K. Sako (Eds.): ASIACRYPT 2012, LNCS 7658, pp. 262-277, 2012.

(C) International Association for Cryptologic Research 2012 
It is the core component of the 3GPP Confidentiality and Integrity Algorithms 128-EEA3 \& 128-EIA3 which were proposed for inclusion in the " $4 \mathrm{G}$ " mobile standard LTE (Long Term Evolution). In July 2010, the ZUC 1.4 [11] was made public for evaluation. We developed two key recovery attacks against ZUC 1.4 [16], and our attacks directly led to the tweak of ZUC 1.4 into ZUC 1.5 [12] in Jan 2011. (Note that it was reported independently in [14] that the non-injective initialization of ZUC 1.4 may result in identical keystreams.) The latest version, ZUC 1.6 [13], was released in June 2011 (ZUC 1.6 and ZUC 1.5 have almost the same specifications).

In this paper, we present the details of our differential attacks against ZUC 1.4. Our attacks against ZUC is similar to the differential attacks against Py, Py6 and Pypy [17], in which different IVs result in identical keystreams. In the first attack against ZUC 1.4, the difference is at the first byte of the IV, and one in $2^{15.4}$ keys results in identical keystreams after testing $2^{13.3}$ IV pairs for each key. Once identical keystreams are detected, the key can be recovered with complexity $2^{99.4}$. In the second attack against ZUC 1.4 , the difference is at the second byte of the IV, and identical keystreams can be obtained after testing $2^{54}$ IVs. The key can be recovered with complexity $2^{67}$.

This paper is organized as follows. The notations and the description of ZUC 1.4 are give in Sect. 2. The overview of the attack is is given in Sect. 3. In Section 4 and 5, we present the key recovery attack with difference at the first byte and the second byte of IV, respectively. We suggest the tweak to fix the flaw in Sect. 6. Section 7 concludes the paper.

\section{Preliminaries}

\subsection{The Notations}

In this paper, we follow the notations used in the ZUC specifications [11].

$\begin{aligned}+ & \text { The addition of two integers } \\ \oplus & \text { The bit-wise exclusive-or operation of integers } \\ \boxplus & \text { The modulo } 2^{32} \text { addition } \\ a b & \text { The product of integers } a \text { and } b \\ a \| b & \text { The concatenation of } a \text { and } b \\ a<<k & \text { The } k \text {-bit cyclic shift of } a \text { to the left } \\ a \gg>k & \text { The } k \text {-bit cyclic shift of } a \text { to the right } \\ a \gg k & \text { The k-bit right shift of integer } a \\ a_{H} & \text { The most significant } 16 \text { bits of integer } a \\ a_{L} & \text { The least significant } 16 \text { bits of integer } a \\ \left(a_{1}, a_{2}, \ldots, a_{n}\right) \rightarrow\left(b_{1}, b_{2}, \ldots, b_{n}\right) & \text { It assigns the values of } a_{i} \text { to } b_{i} \text { in parallel }\end{aligned}$




$$
\begin{array}{cl}
0_{n} & \text { The sequence of } n \text { bits } 0 \\
1_{n} & \text { The sequence of } n \text { bits } 1 \\
\bar{y} & \text { The bitwise complement of } y
\end{array}
$$

An integer $a$ can be written in different formats. For example,

$$
\begin{aligned}
a & =25 & & \text { decimal representation } \\
& =0 x 19 & & \text { hexadecimal representation } \\
& =00011001_{2} & & \text { binary representation }
\end{aligned}
$$

We number the least significant bit with 1 and use $A[i]$ to denote the $i$ th bit of a $A$. And use $B[i . . j]$ to denote the bit $i$ to bit $j$ of $B$.

\subsection{The General Structure of ZUC 1.4}

ZUC is a word-oriented stream cipher with 128-bit secret key and a 128-bit initial vector. It consists of three main components: the linear feedback shift register (LFSR), the bit-reorganization (BR) and a nonlinear function $F$. The general structure of the algorithm is illustrated in Fig. 1.

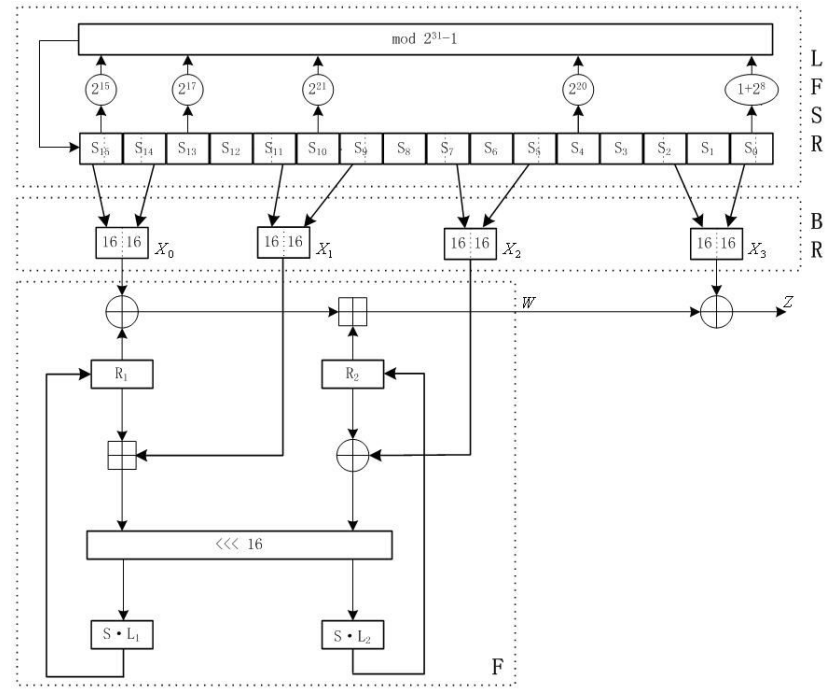

Fig. 1. General structure of ZUC

Linear Feedback Shift Register(LFSR). It consists of sixteen 31-bit registers $s_{0}, s_{1}, \ldots, s_{15}$, and each register is an integer in the range $\left\{1,2, \ldots, 2^{31}-1\right\}$. During the keystream generation stage, the LFSR is updated as follows: 
LFSRUpdate():

1. $s_{16}=\left(2^{15} s_{15}+2^{17} s_{13}+2^{21} s_{10}+2^{20} s_{4}+\left(1+2^{8}\right) s_{0}\right) \bmod \left(2^{31}-1\right)$;

2. If $s_{16}=0$ then set $s_{16}=2^{31}-1$;

3. $\left(s_{1}, s_{2}, \ldots, s_{15}, s_{16}\right) \rightarrow\left(s_{0}, s_{1}, \ldots, s_{14}, s_{15}\right)$.

Bit-Reorganization Function. It extracts 128 bits from the state of the LFSR and forms four 32-bit words $X_{0}, X_{1} X_{2}$ and $X_{3}$ as follows:

Bitreorganization():

1. $X_{0}=s_{15 H} \| s_{14 L}$

2. $\quad X_{1}=s_{11 L}|| s_{9 H}$

3. $\quad X_{2}=s_{7 L} \| s_{5 H}$;

4. $X_{3}=s_{2 L} \| s_{0 H}$

Nonlinear Function $\boldsymbol{F}$. It contains two 32-bit memory words $R_{1}$ and $R_{2}$. The description of $F$ is given below. In function $F, S$ is the Sbox layer and $L_{1}$ and $L_{2}$ are linear transformations as defined in [11]. The output of function $F$ is a 32-bit word $W$. The keystream word $Z$ is given as $Z=W \oplus X_{3}$.

$F\left(X_{0}, X_{1}, X_{2}\right)$ :

1. $W=\left(X_{0} \oplus R_{1}\right) \boxplus R_{2}$

2. $W_{1}=R_{1} \boxplus X_{1}$

3. $W_{2}=R_{2} \oplus X_{2}$;

4. $R_{1}=S\left(L_{1}\left(W_{1 L}|| W_{2 H}\right)\right)$;

5. $R_{2}=S\left(L_{2}\left(W_{2 L}|| W_{1 H}\right)\right)$;

\subsection{The Initialization of ZUC 1.4}

The initialization of ZUC 1.4 consists of two steps: loading the key and IV into the register, and running the cipher for 32 steps with the keystream word being used to update the state.

Key and IV Loading. Denote the 16 key bytes as $k_{i}(0 \leq i \leq 15)$, the $16 \mathrm{IV}$ bytes as $i v_{i}(0 \leq i \leq 15)$. We load the key and IV into the register as: $s_{i}=\left(k_{i}\left\|d_{i}\right\| i v_{i}\right)$. The values of the constants $d_{i}$ are given in [11]. The two memory words $R_{1}$ and $R_{2}$ in function $F$ are set as 0 .

Running the Cipher for 32 Steps. At the initialization stage, the keystream word $Z$ is used to update the LFSR as follows:

LFSRWithInitialisationMode $(u)$ :

1. $v=\left(2^{15} s_{15}+2^{17} s_{13}+2^{21} s_{10}+2^{20} s_{4}+\left(1+2^{8}\right) s_{0}\right) \bmod \left(2^{31}-1\right)$;

2. If $v=0$ then set $v=2^{31}-1$;

3. $s_{16}=v \oplus u$;

4. If $s_{16}=0$ then set $s_{16}=2^{31}-1$;

5. $\left(s_{1}, s_{2}, \ldots, s_{15}, s_{16}\right) \rightarrow\left(s_{0}, s_{1}, \ldots, s_{14}, s_{15}\right)$. 
The cipher runs for 32 steps at the initialization stage as follows:

InitializationStage ():

$$
\text { for } i=0 \text { to } 31\{
$$

1. Bitreorganization();

2. $Z=F\left(X_{0}, X_{1}, X_{2}\right) \oplus X_{3}$;

3. LFSRWithInitialisationMode $(Z \gg 1)$.

\}

\section{Overview of the Attacks}

We notice that the LFSR in ZUC is defined over $G F\left(2^{31}-1\right)$, with the element 0 being replaced with $2^{31}-1$. To the best of our knowledge, it is the first time that $G F\left(2^{31}-1\right)$ is used in the design of stream cipher. In the initialization of ZUC 1.4, we notice that XOR is involved in the update of $\operatorname{LFSR}\left(s_{16}=v \oplus u\right)$. When XOR is applied to the elements in $G F\left(2^{31}-1\right)$, we obtain the following undesirable property:

Property 1. Suppose that $a$ and $a^{\prime}$ are two elements in $G F\left(2^{31}-1\right), a \neq a^{\prime}$, and $\bar{a}=a^{\prime}$. If $b=a$ or $b=\bar{a}$, then $a \oplus b \bmod \left(2^{31}-1\right)=a^{\prime} \oplus b \bmod \left(2^{31}-1\right)=0$.

The above property shows that the difference between $a$ and $a^{\prime}$ can get eliminated with an XOR operation! In the rest of this paper, we exploit this property to attack ZUC 1.4 by eliminating the difference in the state.

In our attacks, we try to eliminate the difference in the state without the difference in the state being injected into the nonlinear function $F$. The reason is that if a difference is injected into $F$, then Sboxes would be involved, and the difference would remain in $F$ until additional difference being injected into $F$, thus the probability that the difference in the state being eliminated would get significantly reduced.

We now investigate what are the IV differences that would result in the difference in the state being eliminated with high probability. The IV differences are classified into the following three types:

Type 1. $\Delta i v_{i} \neq 0$ for at least one value of $i(7 \leq i \leq 15)$.

After loading this type of IVs into LFSR, the difference would appear at the least significant byte of at least one of the LFSR elements $s_{7}, s_{8}, \cdots, s_{15}$. Note that the least significant byte of $s_{7}$ is part of $X_{2}$ in the Bit-reorganization function since $X_{2}=s_{7 L} \| s_{5 H}$, and $X_{2}$ is an input to function $F$. Due to the shift of LFSR, the difference at the least significant byte of $s_{7}, s_{8}, \cdots, s_{15}$ would be injected into $F$. Thus we would not use this type of IV difference in our attacks.

Type 2. $\Delta i v_{i}=0$ for $7 \leq i \leq 15, \Delta i v_{i} \neq 0$ for at least one value of $i(2 \leq i \leq 6)$. After loading this type of IVs into LFSR, the difference would appear at the least 
significant byte of at least one of the LFSR elements $s_{2}, s_{3}, \cdots, s_{6}$. Note that the least significant byte of $s_{2}$ is part of $X_{3}$ in the Bit-reorganization function since $X_{3}=s_{2 L} \| s_{0 H}, X_{3}$ is XORed with the output of $F$ to generate keystream word $Z$, and $Z$ is used to update the LFSR. Two steps later, the difference in $i v_{2}$ would appear in the feedback function to update LFSR. It means that if there is difference in $i v_{2}$, the difference in $s_{2}$ would be used to update the LFSR twice, and the probability that the difference would be eliminated is very small. Due to the shift of LFSR, the difference at $s_{2}, s_{3}, \cdots, s_{7}$ would be eliminated with very small probability. Thus we did not use this type of IV difference in our attacks.

Type 3. $\Delta i v_{i}=0$ for $2 \leq i \leq 15, \Delta i v_{0} \neq 0$ or $\Delta i v_{1} \neq 0$.

The focus of our attacks is on this type of IV differences. In order to increase the chance of success, we consider the difference at only one byte of the IV. We discuss below how the difference in the state can be eliminated when there is difference in $s_{0}$ (the analysis for the difference in $s_{1}$ is similar). At the first step in the initialization,

$$
\begin{aligned}
s_{0} & =\left(k_{0}\left\|d_{0}\right\| i v_{0}\right), \\
v & =2^{15} s_{15}+2^{17} s_{13}+2^{21} s_{10}+2^{20} s_{4}+\left(1+2^{8}\right) s_{0} \quad \bmod \left(2^{31}-1\right), \\
s_{16} & =v \oplus u .
\end{aligned}
$$

Suppose that the difference is only at $i v_{0}$, and $i v_{0}-i v_{0}^{\prime}=\Delta i v_{0}>0$. From (1) and (2) we know that

$$
\begin{aligned}
v-v^{\prime} & =\left(1+2^{8}\right)\left(i v_{0}-i v_{0}^{\prime}\right) \bmod \left(2^{31}-1\right) \\
& =\Delta i v_{0} \| \Delta i v_{0} .
\end{aligned}
$$

If we need to eliminate the difference in $s_{16}$, from Property 1 and (3), the following condition should be satisfied:

$$
\begin{aligned}
v \oplus v^{\prime} & =1_{31} \\
u & =v \quad \text { or } \quad u=v^{\prime}
\end{aligned}
$$

According to (5), $v$ and $v^{\prime}$ have XOR difference in the left-most 15 bits (i.e. $v[17 . .31]$ and $v^{\prime}[17 . .31]$ ), while according to (4), the subtraction difference of those bits are 0 . The only possible reason is that the 15 bits, $v[17 . .31]$, are all affected by the carries from the addition of $\Delta i v_{0}$ to $v^{\prime}$. After testing all the one-byte differences, we found that $v$ must be in one of the following four forms (the values of $v$ and $v^{\prime}$ can be swapped):

$$
\begin{array}{cc} 
& v=111111111111111_{2}\|y\| 1_{2} \| y \\
\text { or } & v=0111111111111111_{2}\|y\| 0_{2} \| y \\
\text { or } & v=0000000000000000_{2}\|\bar{y}\| 0_{2} \| \bar{y} \\
\text { or } & v=1000000000000000_{2}\|\bar{y}\| 1_{2} \| \bar{y} \\
& (y \text { is a 7-bit integer. })
\end{array}
$$


There are 510 possible values of $v\left(v=1_{31}\right.$ and $v=0_{31}$ are excluded since one of $v$ and $\bar{v}$ cannot be 0$)$. All the $\left(v, v^{\prime}\right)$ pairs and their differences are given in Table 1 in Appendix A. Notice that we ignored the order of $v$ and $v^{\prime}$ as they are exchangeable. We have obtained all the possible values of $v$ and $u$ for generating identical keystreams.

We highlight the following property in the table: the difference between $v$ and $v^{\prime}$ uniquely determines the value of pair $\left(v, v^{\prime}\right)$ in the table. As a result, if we know the difference of IVs that results in the collision of the state, we can determine the value of $\left(v, v^{\prime}\right)$ immediately.

By eliminating the difference in the state as illustrated above, we developed two attacks against ZUC 1.4. The first attack is to exploit the difference at $i v_{0}$, and the second attack is to exploit the difference at $i v_{1}$. The details are given in the following two sections.

\section{Attack ZUC 1.4 with Difference at $i v_{0}$}

In this section, we present our first differential attack on the initialization by using IV difference at $i v_{0}$ and generating identical keystream. The keys that generate the same keystream are called weak keys in this attack. We will show that a weak key exists with probability $2^{-15.4}$, and a weak key can be detected with about $2^{13.3}$ chosen IVs. Once a weak key is detected, its effective key size is reduced from 128 bits to around 100 bits.

\subsection{The Weak Keys for $\Delta i v_{0}$}

We will show that when there is difference at $i v_{0}$, about one in $2^{15.4}$ keys would result in identical keystream. For a random key, we will check whether there exists a pair of IVs such that (5), (6) and (7) can be satisfied.

We start with analyzing how keys and IVs are involved in the expression of $u$ and $v$ in the first step of initialization. From the specifications of the initialization, we have

$$
\begin{aligned}
u & =Z \gg 1=\left(X_{0} \oplus X_{3}\right) \gg>1=\left(\left(s_{15 H} \| s_{14 L}\right) \oplus\left(s_{2 L} \| s_{0 H}\right)\right) \gg>1 \\
& =\left(\left(k_{15}\left\|i v_{2}\right\| k_{0} \| i v_{14}\right) \oplus 0 \mathrm{x} 6 \mathrm{~b} 8 \mathrm{f} 9 \mathrm{a} 89\right) \gg>1
\end{aligned}
$$

In (2) and (8), there are 5 bytes of key, $\left\{k_{0}, k_{4}, k_{10}, k_{13}, k_{15}\right\}$, and 7 bytes of IV, $\left\{i v_{0}, i v_{2}, i v_{4}, i v_{10}, i v_{13}, i v_{14}, i v_{15}\right\}$ being involved in the computation of $u$ and $v$. The complexity would be very high if we directly try all possible combinations of the keys and IVs. However, with analysis on the expressions of $u$ and $v$, we can reduce the search space from $2^{96}$ to around $2^{26.3}$. 
Solve (5), (6), (7) and (8), we obtain the following four groups of solutions:

Group 1.

$$
\begin{aligned}
u & =v=1111111111111111_{2}\|y\| 1_{2} \| y \\
k_{15} & =0 \mathrm{x} 94 \\
i v_{2} & =0 \mathrm{x} 70 \\
k_{0} & =0 \mathrm{x} 9 \mathrm{a} \oplus\left(y \| 1_{2}\right) \\
i v_{14}>>1 & =0 \mathrm{x} 44 \oplus y
\end{aligned}
$$

Group 2.

$$
\begin{aligned}
u & =v=0111111111111111_{2}\|y\| 0_{2} \| y \\
k_{15} & =0 \mathrm{x} 14 \\
i v_{2} & =0 \mathrm{x} 70 \\
k_{0} & =0 \mathrm{x} 9 \mathrm{a} \oplus\left(y \| 0_{2}\right) \\
i v_{14}>1 & =0 \mathrm{x} 44 \oplus y
\end{aligned}
$$

Group 3.

$$
\begin{aligned}
u & =v=0000000000000000_{2}\|\bar{y}\| 0_{2} \| \bar{y} \\
k_{15} & =0 \mathrm{x} 6 \mathrm{~b} \\
i v_{2} & =0 \mathrm{x} 8 \mathrm{f} \\
k_{0} & =0 \mathrm{x} 9 \mathrm{a} \oplus\left(\bar{y} \| 0_{2}\right) \\
i v_{14} \gg 1 & =0 \mathrm{xbb} \oplus \bar{y}
\end{aligned}
$$

Group 4.

$$
\begin{aligned}
u & =v=1000000000000000_{2}\|\bar{y}\| 1_{2} \| \bar{y} \\
k_{15} & =0 \text { xeb } \\
i v_{2} & =0 x 8 \mathrm{f} \\
k_{0} & =0 x 9 \mathrm{a} \oplus\left(\bar{y} \| 1_{2}\right) \\
i v_{14}>>1 & =0 x b b \oplus \bar{y}
\end{aligned}
$$

Furthermore, from (2) we compute $v$ as follows (note that the property $2^{k} s_{i}$ $\left.\bmod \left(2^{31}-1\right)=s_{i}<<k\right)$ :

$$
\begin{aligned}
v= & \left(1+2^{23}\right) k_{0}+2^{7} k_{15}+2^{9}\left(k_{13}+2^{3} k_{4}+2^{4} k_{10}\right)+\left(1+2^{8}\right) i v_{0} \\
& +2^{15}\left(i v_{15}+2^{2} i v_{13}+2^{5} i v_{4}+2^{6} i v_{10}\right)+0 \mathrm{x} 451 \mathrm{bfe} 1 \mathrm{~b} \bmod \left(2^{31}-1\right)
\end{aligned}
$$

Let $s_{1} m_{1}=k_{13}+2^{3} k_{4}+2^{4} k_{10}, s u m_{2}=i v_{15}+2^{2} i v_{13}+2^{5} i v_{4}+2^{6} i v_{10}$. The value of sum $_{1}$ ranges from 0 to 6375, and the value of sum $_{2}$ ranges from 0 to 25755 . We developed Algorithm 1 to search for weak keys. 


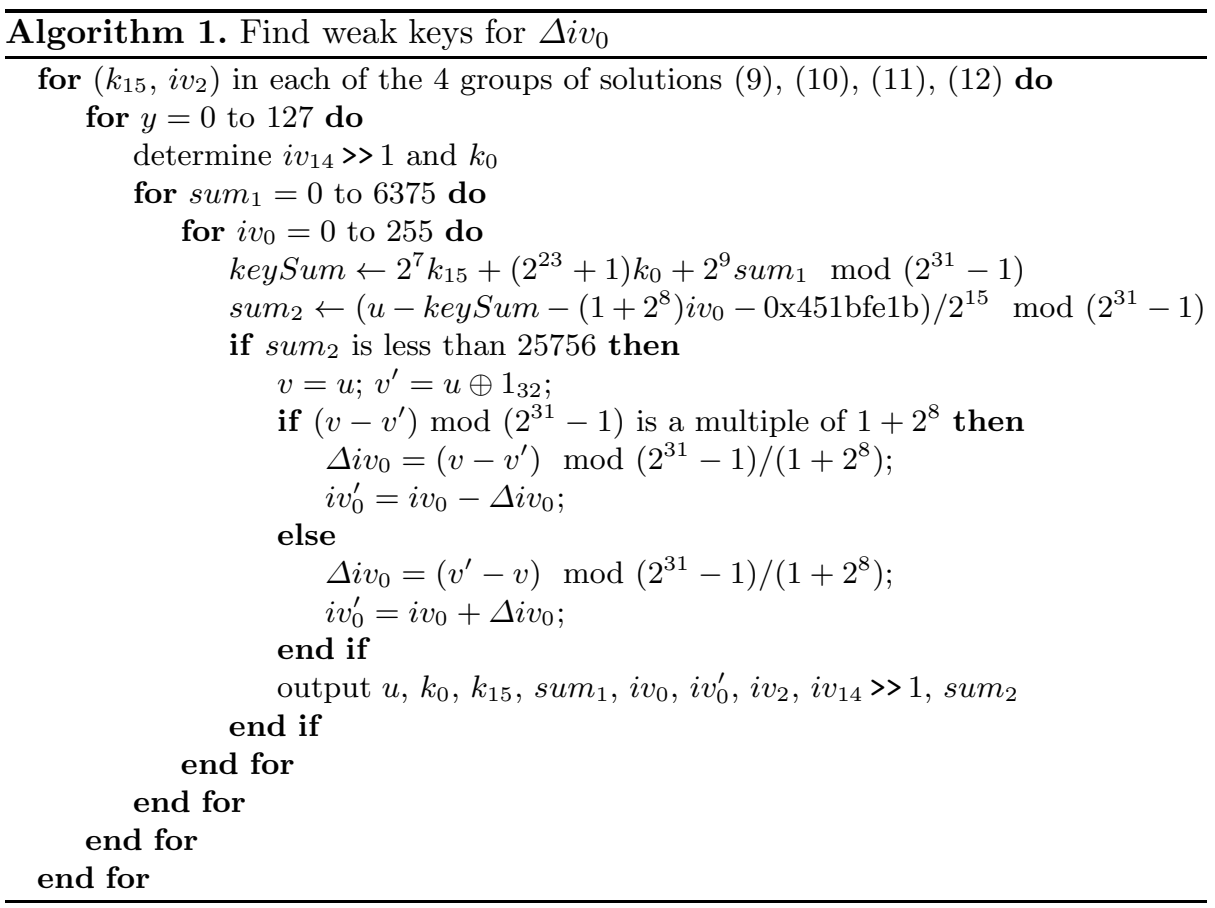

Each output from Algorithm 1 gives the value of $\left(k_{15}, k_{0}, s u m_{1}, i v_{0}, i v_{0}^{\prime}\right.$, $\left.i v_{2}, i v_{14}, s_{2} m_{2}\right)$ that results in identical keystreams. Running Algorithm 1, we found $9934=2^{13.28}$ different outputs. We note that on average, each sum $_{1}$ from the output of the algorithm represents $2^{24} / 6376=2^{11.36}$ possible choices of $\left(k_{4}, k_{10}, k_{13}\right)$. Thus there are $2^{13.3} \times 2^{11.4}=2^{24.7}$ weak values of $\left(k_{0}, k_{4}, k_{10}, k_{13}, k_{15}\right)$. Hence, there are $2^{24.7}$ weak keys out of $2^{40}$ possible values of the 5 key bytes. The probability that a random key is weak for IV difference at $i v_{0}$ is $2^{-15.4}$. The complexity of Algorithm 1 is $4 \times 128 \times 6376 \times 256=2^{26.3}$.

Identical Keystreams. We give below a weak key and an IV pair with difference at $i v_{0}$ that result in identical keystreams.

$$
\begin{aligned}
k e y & =87,4,95,13,161,32,199,61,20,147,56,84,126,205,165,148 \\
I V & =166,166,112,38,192,214,34,211,170,25,18,71,4,135,68,5 \\
I V^{\prime} & =116,166,112,38,192,214,34,211,170,25,18,71,4,135,68,5
\end{aligned}
$$

For both $I V$ and $I V^{\prime}$, the identical keystreams are: 0xbfe800d5 0360a22b 6c4554c8 67f00672 2ce94f3f f94d12ba 11c382b3 cbaf4b31....

\subsection{Detecting Weak Keys for $\Delta i v_{0}$}

We have shown above that a random key is weak with probability $2^{-15.4}$. In the attack against ZUC, we will first detect a weak key, then recover it. To detect 
a weak key, our approach is to use the IV pairs generated from Algorithm 1 to test whether identical keystreams are generated. Note that for a particular value of $s u m_{2}$, we can always find a combination of $\left(i v_{4}, i v_{10}, i v_{13}, i v_{15}\right\}$ that satisfies sum $_{2}=i v_{15}+2^{2} i v_{13}+2^{5} i v_{4}+2^{6} i v_{10}$. Thus a pair of IVs $\left(i v_{0}, i v_{2}, i v_{4}, i v_{10}\right.$, $\left.i v_{13}, i v_{14}, i v_{15}\right)$ and $\left(i v_{0}^{\prime}, i v_{2}, i v_{4}, i v_{10}, i v_{13}, i v_{14}, i v_{15}\right)$ can be determined by each output of Algorithm 1. Using this result, we developed Algorithm 2 to detect weak keys for $\Delta i v_{0}$.

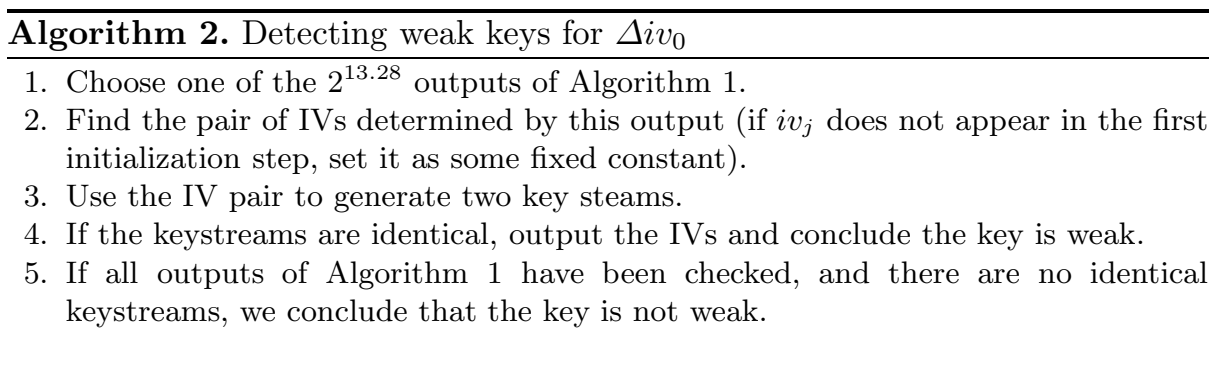

In Algorithm 2, we need to test at most $2^{13.3}$ pairs of IVs to determine if a key is weak for difference at $i v_{0}$.

\subsection{Recovering Weak Keys for $\Delta i v_{0}$}

After detecting a weak key, we proceed to recover the weak key. Once a key is detected as weak (as given from Algorithm 2), from the IV pair being used to generate identical keystreams, we immediately know the value of $k_{0}, k_{15}$ and $s u m_{1}$. Note that $s u m_{1}=\left(k_{13}+2^{3} k_{4}+2^{4} k_{10}\right)$. In the best situations, the sum is 0 or 25755 , then we can uniquely determine $k_{4}, k_{10}$ and $k_{13}$. In the worst situation, there are $2^{12}$ possible choices for $k_{4}, k_{10}$ and $k_{13}$, and therefore, we need $2^{12}$ tests to determine the correct values for $k_{4}, k_{10}$ and $k_{13}$. On average, for each value of $s u m_{1}$, we need to test $2^{11.4}$ combinations of $\left(k_{4}, k_{10}, k_{13}\right)$.

Since there are only five key bytes being recovered in our attack, the remaining 11 key bytes should be recovered with exhaustive search. Hence, the complexity to recover all key bits is $2^{88} \times 2^{11.4}=2^{99.4}$. From the analysis above, we also know that the best complexity is $2^{88}$ and the worst complexity is $2^{100}$.

\section{Attack ZUC 1.4 with Difference at $i v_{1}$}

In this section, we present the differential attack on ZUC 1.4 for IV difference at $i v_{1}$. Different from the attack in Section 4, we need to consider the computation of $u$ and $v$ in the second step of the initialization. For this type of IV difference, for every key, there are some IV pairs that result in identical keystreams since more IV bytes are involved. Once we found such an IV pair, we can recover the key with complexity around $2^{67}$. 


\subsection{Identical Keystreams for $\Delta i v_{1}$}

The computation of $u$ and $v$ in the second initialization step involves more key and IV bytes. The $v$ in the second initialization step is computed as:

$$
\begin{aligned}
v= & \left(2^{15} s_{16}+2^{17} s_{14}+2^{21} s_{11}+2^{20} s_{5}+\left(1+2^{8}\right) s_{1}\right) \bmod \left(2^{31}-1\right), \\
s_{16}= & \left(\left(2^{15} s_{15}+2^{17} s_{13}+2^{21} s_{10}+2^{20} s_{4}+\left(1+2^{8}\right) s_{0}\right) \bmod \left(2^{31}-1\right)\right) \\
& \oplus\left(\left(\left(k_{15}\left\|i v_{2}\right\| k_{0} \| i v_{14}\right) \oplus 0 \mathrm{x} 6 \mathrm{~b} 8 \mathrm{f} 9 \mathrm{a} 89\right)>>1\right)
\end{aligned}
$$

And $u$ is given as:

$$
\begin{aligned}
& u=\left(\left(\left(X_{0} \oplus R_{1}\right)+R_{2}\right) \oplus X_{3}\right) \gg>1 \\
& X_{0}=\left(s_{16 H}\left\|10101100_{2}\right\| i v_{15}\right) \\
& X_{3}=\left(01011110_{2}\left\|i v_{3}\right\| k_{1} \| 01001101_{2}\right) \\
& R_{1}=S\left(L_{1}\left(s_{9 H} \| s_{7 L}\right)\right)=f_{1}\left(i v_{7}, k_{9}\right) \\
& R_{2}=S\left(L 2\left(s_{5 H} \| s_{11 L}\right)\right)=f_{2}\left(i v_{11}, k_{5}\right)
\end{aligned}
$$

where $f_{1}$ and $f_{2}$ are some deterministic non-linear functions.

There are $10 \mathrm{IV}$ bytes involved in the expression of $v$, i.e. $\left(i v_{0}, i v_{1}, i v_{2}, i v_{4}, i v_{5}\right.$, $\left.i v_{10}, i v_{11}, i v_{13}, i v_{14}, i v_{15}\right)$ and 8 IV bytes involved in the expression of $u$, i.e. $\left(i v_{0}\right.$, $\left.i v_{3}, i v_{4}, i v_{7}, i v_{10}, i v_{11}, i v_{13}, i v_{15}\right)$. In total, there are 12 IV bytes being involved in the computation of $u$ and $v$, and every bit of $u$ and $v$ can be affected by IV. We conjecture that for every key, the conditions (5) and (6) can be satisfied, and identical keystreams can be generated. To verify it, we tested 1000 random keys. Our experimental results show that there is always an IV pair for each key that results in identical keystreams.

In the attack, a random key and a random iv pair with difference at $i v_{1}$, the probability that $v$ and $u$ satisfy the conditions (5) and (6) is $2^{-31} \times 2^{-31} \times 2=2^{-61}$. Choosing $2^{8} i v$ s with difference at $i v_{1}$, we have around $2^{15}$ pairs. The identical keystream pair appears with probability $2^{-61+15}=2^{-46}$ with $2^{8}$ IVs. We thus need about $2^{46} \times 2^{8}=2^{54}$ IVs to obtain identical keystreams.

Identical Keystreams. We give below a key and an IV pair with difference at $i v_{1}$ that result in identical keystreams. The algorithm being used to find the IV pair is given in Appendix B. The algorithm is a bit complicated since a number of optimization tricks are involved. The explanation of the optimization details is omitted here since our focus is to develop a key recovery attack.

$$
\begin{aligned}
k e y & =123,149,193,87,42,150,117,4,209,101,85,57,46,117,49,243 \\
I V & =92,80,241,10,0,217,47,224,48,203,0,45,204,0,0,17 \\
I V^{\prime} & =92,182,241,10,0,217,47,224,48,203,0,45,204,0,0,17
\end{aligned}
$$

The identical keystreams are: 0xf09cc17d 41f12d3f 453ac0c3 cadcef9f f98fb964 ca6e576e b48b813 6c43da22 .... 


\subsection{Key Recovery for $\Delta i v_{1}$}

After identical keystreams are generated from an IV pair with difference at $i v_{1}$, we proceed to recover the secret key. From Table 1 in Appendix $\mathrm{A}$ we know the value of $\left(v, v^{\prime}\right)$ since we know the difference at $i v_{1}$ of the chosen IV pair, and we also know the value of $u$ since $u=v$ or $u=v^{\prime}$. In the following, we illustrate a key recovery attack after identical keystreams have been detected.

1. In the expression of $u$ in (15), $\left(k_{1}, k_{5}, k_{9}, s_{16 H}\right)$ is involved. Note that there are only two possible values of the 31-bit $u$. We try all the possible values of $\left(k_{1}, k_{5}, k_{9}, s_{16 H}\right)$, then there would be $2^{8 \times 3+16} \times 2^{-31} \times 2=2^{10}$ possible values of $\left(k_{1}, k_{5}, k_{9}, s_{16 H}\right)$ that generate the two possible values of $u$. The complexity of this step is $2^{40}$.

2. Next we use the expression of $s_{16}$ in (14). For each of the $2^{10}$ possible values of $\left(k_{1}, k_{5}, k_{9}, s_{16 H}\right)$, we try all the possible values of $\left(k_{0}, k_{4}, k_{10}, k_{13}, k_{15}\right)$ and check whether the values of $s_{16 H}$ is computed correctly or not. There would be $2^{8 \times 5} \times 2^{-16}=2^{24}$ possible values of $\left(k_{0}, k_{4}, k_{10}, k_{13}, k_{15}\right)$ left. Considering that there are $2^{10}$ possible values of $\left(k_{1}, k_{5}, k_{9}, s_{16 H}\right)$, about $2^{10} \times 2^{24}=2^{34}$ possible values of $\left(k_{0}, k_{1}, k_{4}, k_{5}, k_{9}, k_{10}, k_{13}, k_{15}, s_{16 H}\right)$ remain. The complexity of this step is $2^{8 \times 5} \times 2^{10}=2^{50}$.

3 . Then we use the expression of $v$ in (14). For each of the $2^{34}$ possible values of $\left(k_{0}, k_{1}, k_{4}, k_{5}, k_{9}, k_{10}, k_{13}, k_{15}, s_{16 H}\right)$, we try all the possible values of $\left(k_{11}, k_{14}\right)$ and check whether the value of $v$ is correct or not. A random value of $\left(k_{11}, k_{14}\right)$ would pass the test with probability $2^{8 \times 2} \times 2^{-31}=2^{-15}$ Considering that there are $2^{34}$ possible values of $\left(k_{0}, k_{1}, k_{4}, k_{5}, k_{9}, k_{10}, k_{13}\right.$, $\left.k_{15}, s_{16 H}\right)$, about $2^{34} \times 2^{-15}=2^{19}$ possible values of $\left(k_{0}, k_{1}, k_{4}, k_{5}, k_{9}, k_{10}\right.$, $\left.k_{11}, k_{13}, k_{14}, k_{15}\right)$ remain. The complexity of this step is $2^{8 \times 2} \times 2^{34}=2^{50}$.

4. For each of the $2^{19}$ possible values of $\left(k_{0}, k_{1}, k_{4}, k_{5}, k_{9}, k_{10}, k_{11}, k_{13}, k_{14}\right.$, $\left.k_{15}\right)$, we recover the remaining 6 key bytes $\left(k_{2}, k_{3}, k_{6}, k_{7}, k_{8}, k_{12}\right)$ by exhaustive search. The complexity of this step is $2^{19} \times 2^{8 \times 6}=2^{67}$.

The overall computational complexity to recover a key is $2^{40}+2^{50}+2^{50}+2^{67} \approx$ $2^{67}$. And we need about $2^{54}$ IVs in the attack. Note that the complexity in the first, second and third steps can be significantly reduced with optimization since we are dealing with simple functions. For example, meet-in-the-middle attack can be used in the first step, and the sum of a few key bytes can be considered in the second and third steps. However, the complexity of those three steps has little effect on the overall complexity of the attack, so we do not present the details of the optimization here.

\section{Improving ZUC 1.4}

From the analysis in Sect. 3, the weakness of the initialization comes from the non-injective update of the LFSR. To fix the flaw, we proposed the tweak in the rump session of Asiacrypt 2010. Instead of using the XOR operation, it is better to use addition modulo operation over $G F\left(2^{31}-1\right)$. More specifically, 
the operation $s_{16}=v \oplus u$ is changed to $s_{16}=v+u \bmod \left(2^{31}-1\right)$. With this tweak, the difference in $v$ would always result in the difference in $s_{16}$ if there is no difference in $u$, and the attack against ZUC 1.4 can no longer be applied. In the later versions ZUC 1.5 and 1.6 (ZUC 1.5 and 1.6 have almost the same specifications), the computation of $s_{16}$ is modified using our suggested method.

\section{Conclusion}

In this paper, we developed two chosen IV attacks against the initialization of ZUC 1.4. In our attacks, identical keystreams are generated from different IVs, then key recovery attacks are applied. Our attacks are independent of the number of steps in initialization. The lesson from this paper is that when non-injective functions are used in cipher design, we should pay special attention to ensure that the difference cannot be eliminated with high probability.

\section{References}

1. Babbage, S., Dodd, M.: The MICKEY Stream Ciphers. In: Robshaw, M., Billet, O. (eds.) New Stream Cipher Designs. LNCS, vol. 4986, pp. 191-209. Springer, Heidelberg (2008)

2. Berbain, C., Billet, O., Canteaut, A., Courtois, N.T., Gilbert, H., Goubin, L., Gouget, A., Granboulan, L., Lauradoux, C., Minier, M., Pornin, T., Sibert, H.: Sosemanuk, a Fast Software-Oriented Stream Cipher. In: Robshaw, M., Billet, O. (eds.) New Stream Cipher Designs. LNCS, vol. 4986, pp. 98-118. Springer, Heidelberg (2008)

3. Bernstein, D.J.: The Salsa20 Family of Stream Ciphers. In: Robshaw, M., Billet, O. (eds.) New Stream Cipher Designs. LNCS, vol. 4986, pp. 84-97. Springer, Heidelberg (2008)

4. Biryukov, A., Shamir, A., Wagner, D.: Real Time Cryptanalysis of A5/1 on a PC. In: Schneier, B. (ed.) FSE 2000. LNCS, vol. 1978, pp. 1-44. Springer, Heidelberg (2001)

5. Boesgaard, M., Vesterager, M., Zenner, E.: The Rabbit Stream Cipher. In: Robshaw, M., Billet, O. (eds.) New Stream Cipher Designs. LNCS, vol. 4986, pp. 69-83. Springer, Heidelberg (2008)

6. De Cannière, C., Preneel, B.: Trivium. In: Robshaw, M., Billet, O. (eds.) New Stream Cipher Designs. LNCS, vol. 4986, pp. 244-266. Springer, Heidelberg (2008)

7. Fluhrer, S., Mantin, I., Shamir, A.: Weaknesses in the Key Scheduling Algorithm of RC4. In: Vaudenay, S., Youssef, A.M. (eds.) SAC 2001. LNCS, vol. 2259, pp. 1-24. Springer, Heidelberg (2001)

8. Golić, J.D.: Cryptanalysis of Alleged A5 Stream Cipher. In: Fumy, W. (ed.) EUROCRYPT 1997. LNCS, vol. 1233, pp. 239-255. Springer, Heidelberg (1997)

9. Hell, M., Johansson, T., Maximov, A., Meier, W.: The Grain Family of Stream Ciphers. In: Robshaw, M., Billet, O. (eds.) New Stream Cipher Designs. LNCS, vol. 4986, pp. 179-190. Springer, Heidelberg (2008)

10. Rivest, R.L.: The RC4 Encryption Algorithm. RSA Data Security, Inc. (March 1992) 
11. ETSI/SAGE Specification. Specification of the 3GPP Confidentiality and Integrity Algorithms 128-EEA3 \& 128-EIA3. Document 2: ZUC Specification; Version: 1.4 (July 30, 2010)

12. ETSI/SAGE Specification. Specification of the 3GPP Confidentiality and Integrity Algorithms 128-EEA3 \& 128-EIA3. Document 2: ZUC Specification; Version: 1.5 (January 4, 2011)

13. ETSI/SAGE Specification. Specification of the 3GPP Confidentiality and Integrity Algorithms 128-EEA3 \& 128-EIA3. Document 2: ZUC Specification; Version: 1.6 (June 28, 2011)

14. Sun, B., Tang, X., Li, C.: Preliminary Cryptanalysis Results of ZUC. In: First International Workshop on ZUC Algorithm, vol. 12 (2010)

15. Wu, H.: The Stream Cipher HC-128. In: Robshaw, M., Billet, O. (eds.) New Stream Cipher Designs. LNCS, vol. 4986, pp. 39-47. Springer, Heidelberg (2008)

16. Wu, H., Nguyen, P.H., Wang, H., Ling, S.: Cryptanalysis of the Stream Cipher ZUC in the 3GPP Confidentiality \& Integrity Algorithms 128-EEA3 \& 128-EIA3. In: Rump Session of Asiacrypt 2010 (2008)

17. Wu, H., Preneel, B.: Differential Cryptanalysis of the Stream Ciphers Py, Py6 and Pypy. In: Naor, M. (ed.) EUROCRYPT 2007. LNCS, vol. 4515, pp. 276-290. Springer, Heidelberg (2007) 


\section{A The List of Possible $v$ and $v^{\prime}$ for Collision}

Table 1. The list of possible $v, v^{\prime}$

\begin{tabular}{|c|c|c|c|c|c|c|c|c|c|c|c|}
\hline ndex & & $v^{\prime}$ & $\Delta i v$ & Index & & & $\Delta i v$ & Index & & & $\Delta i v$ \\
\hline 1 & $0 x 3 f f f 8000$ & $8 x 40007 f f f$ & $\overline{0 x f f}$ & 86 & $0 x 3 f f f$ & $0 x 40002 a a a$ & $0 x 55$ & 171 & $0 x 7 f f f a a a a$ & $0 \times 5555$ & \\
\hline 2 & $x 3 f f f 8101$ & $0 x 40007$ efe & $0 x f d$ & 87 & $0 x 3 f f f d 656$ & $0 \times 400029 a 9$ & $0 x 53$ & 172 & $x 7 f f f a b a b$ & $x 5454$ & \\
\hline 3 & $x 3 f f f \varepsilon$ & $0 \times 40007 d f d$ & $0 x f b$ & 88 & $x 3 f f f d 757$ & $x 400028 a 8$ & $0 x 51$ & 173 & $\overline{x 7 f f f a c a c}$ & $x 5353$ & $\overline{0 x a 6}$ \\
\hline 4 & $0 x 3 f f f 8303$ & $0 x 40007 c f c$ & $0 x f 9$ & 89 & $0 x 3 f f f d 858$ & $0 \times 400027 a 7$ & $0 x 4 f$ & 174 & $\overline{0 x 7 f f f a d a d}$ & $0 x 5252$ & $0 x a 4$ \\
\hline 5 & $0 \times 3 f f f 8404$ & $0 \times 40007 \mathrm{bfb}$ & $0 x f 7$ & 90 & $0 x 3 f f f d 959$ & $0 \times 400026 a 6$ & $0 \times 4 d$ & 175 & $0 x 7 f f f a e a e$ & & $0 x a 2$ \\
\hline 6 & $0 x 3 f f f 8505$ & $0 \times 40007 a f a$ & $0 x f 5$ & 91 & $0 \times 3 f f f d a 5 a$ & $0 \times 400025 a 5$ & $0 \times 4 b$ & 176 & $0 x 7 f f f a f a f$ & $0 \times 5050$ & \\
\hline 7 & $0 x 3 f f f 8606$ & $0 \times 400079 f 9$ & $0 x f 3$ & 92 & $0 x 3 f f f d b 5 b$ & $0 \times 400024 a 4$ & $0 x 49$ & 177 & $0 x 7 f f f b 0 b 0$ & $x 4 f 4 f$ & \\
\hline 8 & $0 \times 3 f f f 8707$ & $0 \times 400078 f 8$ & $0 x f 1$ & 93 & $0 x 3 f f f d c 5 c$ & $5 x 400023 a 3$ & $0 \times 47$ & 178 & $x 7 f f f b 1 b 1$ & $x 4 e 4 e$ & $0 x 9 c$ \\
\hline 9 & & $0 \times 400077 f 7$ & $0 x e f$ & 94 & $0 \times 3 f f f f d d 5 d$ & $0 x 400022 a 2$ & $0 \times 45$ & 179 & & $9 x 4 d 4 d$ & $0 x 9 a$ \\
\hline 10 & $0 \times 3 f f f 8909$ & $0 \times 400076 f 6$ & oxed & 95 & $0 \times 3 f f f d e 5 e$ & $0 x 400021 a 1$ & $0 x 43$ & 180 & $0 x 7 f f f b 3 b 3$ & & \begin{tabular}{|l|l|}
$0 x 98$ \\
\end{tabular} \\
\hline 11 & $0 x 3 f f f 8 a 0 a$ & $0 \times 400075 f 5$ & $0 x e b$ & 96 & $0 x 3 f f f d f 5 f$ & $0 \times 400020 a 0$ & $0 \times 41$ & 181 & $0 x 7 f f f b 4 b 4$ & $0 \times 4 b 4 b$ & $0 x 96$ \\
\hline 12 & $0 x 3 f f f 8 b 0 b$ & $0 \times 400074 f 4$ & $0 x e 9$ & 97 & $0 x 3 f f f e 060$ & $0 x 40001 f 9 f$ & $0 x 3 f$ & 182 & $0 x 7 f f f b 5 b 5$ & $0 \times 4 a 4 a$ & $\mid 0 x 94$ \\
\hline 13 & $0 x 3 f f f 8 c 0 c$ & $0 x 400073 f 3$ & $0 x e 7$ & 98 & $0 \times 3 f f f e 161$ & $0 x 40001 e 9 e$ & $0 x 3 d$ & 183 & $0 x 7 f f f b 6 b 6$ & $\times 4949$ & $0 x 92$ \\
\hline 14 & $0 x 3 f f f 8 d 0 d$ & & $0 x e 5$ & 99 & $0 x 3 f f f e 262$ & & $0 \times 3 b$ & 184 & & & $0 x 90$ \\
\hline & & & $0 x e 3$ & 100 & & & $0 x 39$ & 185 & $0 x 7 f f f b 8 b 8$ & & $0 x 8 e$ \\
\hline 16 & $0 x 3 f f f 8 f 0 f$ & $0 \times 400070 \mathrm{f0}$ & $0 x e 1$ & 101 & $0 \times 3 f f f e 464$ & $0 \times 40001 b 9 b$ & $0 \times 37$ & 186 & $0 x 7 f f f b 9 b 9$ & $0 \times 4646$ & $0 x 8 c$ \\
\hline 17 & $0 \times 3 f f f 9010$ & $0 x 40006 \mathrm{fef}$ & $0 x d f$ & 102 & $0 x 3 f f f e 565$ & $0 x 40001 a 9 a$ & $0 x 35$ & 187 & $0 x 7 f f f b a b a$ & $0 \times 4545$ & $0 x 8 a$ \\
\hline 18 & $0 x 3 f f f 9$ & $0006 e e=$ & $0 x d d$ & 103 & fffe 666 & 1999 & $x 33$ & 188 & $\overline{b b b}$ & $x 4444$ & \\
\hline 19 & $0 \times 3 f f f 9212$ & $6 \mathrm{ded}$ & $0 x d b$ & 104 & & & $0 \times 31$ & 189 & & & $x 86$ \\
\hline 20 & $0 x 3 f f f 9313$ & & & 105 & & $0 \times 40001797$ & $0 \times 2 f$ & 190 & $0 x 7 f f f b d b d$ & & \begin{tabular}{|l}
$0 x 84$ \\
\end{tabular} \\
\hline 21 & $0 \times 3 f f f 9414$ & $0 x 40006 b e b$ & \begin{tabular}{|l}
$0 x d 7$ \\
\end{tabular} & 106 & $0 x 3 f f f e 969$ & $0 \times 40001696$ & $0 \times 2 d$ & 191 & $0 x 7 f f f b e b e$ & $0 \times 4141$ & $0 x 82$ \\
\hline$\overline{22}$ & $0 \times 3 f f f 9515$ & $\overline{0 x 40006 a e a}$ & $\begin{array}{l}0 x d 5 \\
0\end{array}$ & $\begin{array}{ll}107 \\
\end{array}$ & $0 x 3 f f f$ fea $6 a$ & $0 \times 40001595$ & $0 x_{22 b}$ & 192 & $\overline{0 x 7 f f f b f b f}$ & $\overline{0 x 4040}$ & $0 x 80$ \\
\hline 23 & $0 x 3 f f f 9616$ & $9 e 9$ & $5 x d 3$ & 108 & $\overline{f f f e b 6 b}$ & 1494 & & 193 & & & \\
\hline 24 & $0 x 3 f f f$ & & $\overline{0 x d 1}$ & 109 & & & $0 \times 27$ & 194 & & & $0 x 7 c$ \\
\hline 25 & $0 x 3 f f f 9818$ & $0 \times 400067 e 7$ & $0 x c f$ & 110 & $0 x 3 f f f e d 6 d$ & & $0 \times 25$ & 195 & $0 x 7 f f f c 2 c 2$ & $0 x 3 d 3 d$ & $0 x 7 a$ \\
\hline 26 & $0 \times 3 f f f 9919$ & $0 \times 400066 e 6$ & $0 x c d$ & 111 & $0 \times 3 f f f e e 6 e$ & $0 \times 40001191$ & $0 \times 23$ & 196 & $0 x 7 f f f c 3 c 3$ & $0 \times 3 c 3 c$ & $0 \times 78$ \\
\hline 27 & $0 x 3 f f f 9 a 1 a$ & $0 \times 400065 e 5$ & $0 x c b$ & 112 & $0 x 3 f f f e f 6 f$ & $0 \times 40001090$ & $0 \times 21$ & 197 & $0 x 7 f f f c 4 c 4$ & $0 x 3 b 3 b$ & $0 \times 76$ \\
\hline 28 & $0 x 3 f f f 9 b 1 b$ & & $0 x c 9$ & 113 & & & & & & & \\
\hline 29 & $0 x 3 f f f s$ & & $0 x \subset 7$ & 114 & & & $0 x 1 d$ & 199 & & & $5 x 72$ \\
\hline 30 & $0 x 3 f f f 9 d 1 d$ & $0 \times 40$ & $0 x c 5$ & 115 & $0 x 3 f f f f 272$ & $\overline{0 d 8 d}$ & $0 \times 1 b$ & 200 & $0 x 7 f f f c 7 c 7$ & $0 \times 3838$ & $0 x 70$ \\
\hline 31 & $0 \times 3 f f f 9 e 1 e$ & $0 \times 400061 e 1$ & $0 x c 3$ & 116 & $0 x 3 f f$ & $000 c 8 c$ & $0 \times 19$ & 201 & $0 x 7 f f$ & $0 \times 3737$ & $0 x 6 e$ \\
\hline 32 & $0 x 3 f f f f 9 f 1 f$ & $0 \times 400060 e 0$ & $0 x c 1$ & 117 & $0 x 3 f f f f 474$ & $0 \times 40000 b 8 b$ & $0 \times 17$ & 202 & $0 x 7 f f f c 9 c 9$ & $0 \times 3636$ & $0 x 6 c$ \\
\hline 33 & $0 x 3 f f f a 020$ & $f d f$ & $0 x b f$ & 118 & $0 \times 3 f f f f 575$ & $0 x 40000 a 8 a$ & & 203 & & & $0 \times 6 a$ \\
\hline 34 & $0 x 3 f f f c$ & & $0 x b d$ & 119 & J1070 & & $0 x 13$ & 204 & $b c b$ & & \\
\hline 35 & $0 x 3 f f f a 222$ & $\overline{0 x 4}$ & $0 x b b$ & 120 & & 888 & $0 \times 11$ & 205 & $0 x 7 f f f c c c c$ & & $0 x 66$ \\
\hline 36 & $0 x 3 f f f a 323$ & $0 \times 40$ & $0 \times 69$ & 121 & $f 878$ & $0 \times 40000787$ & $0 x f$ & 206 & $0 x 7 f f f c d c d$ & $0 \times 3232$ & $0 \times 64$ \\
\hline 37 & $0 x 3 f f f a 424$ & $0 \times 4000$ & $0 x b 7$ & 122 & $0 x 3 f f f f 979$ & $0 \times 40000686$ & $0 x d$ & 207 & $0 \times 7 f f f c e c e$ & $0 \times 3131$ & $0 \times 62$ \\
\hline 38 & $0 x 3 f f f a 525$ & $\overline{a d a}$ & $0 x b 5$ & 123 & $\overline{f f f f a 7 a}$ & 000585 & $0 x b$ & 208 & $x 7 f f f c f c f$ & & $0 \times 60$ \\
\hline 39 & $0 x 3 f f f c$ & & $0 x b 3$ & 124 & $f f f b 7 b$ & & $0 \times 9$ & 209 & $\overline{0 d 0}$ & & \\
\hline 40 & $0 \times 3 f f f a 727$ & $0 \times 4$ & $0 x b 1$ & 125 & $x 3 f f f f c 7 c$ & 383 & $0 \times 7$ & 210 & $0 x 7 f f$ & $x 2 e 2 e$ & $0 x 5 c$ \\
\hline 41 & $0 x 3 f f f a 828$ & $8 d 7$ & $0 x a f$ & 126 & $f f f f d$ & 0282 & $0 \times 5$ & 211 & & & $0 \times 5 a$ \\
\hline 42 & $0 x 3 f f f$ & $8 d 6$ & $0 x a d$ & 127 & fe7e & 0181 & $0 \times 3$ & 212 & $0 x 7 f f f d 3 d 3$ & $x 2 c 2 c$ & $0 \times 58$ \\
\hline 43 & $0 x 3$ & & $0 x a b$ & 128 & & & $\begin{array}{ll}0 x 1 \\
\end{array}$ & 213 & $4 d 4$ & & $0 \times 56$ \\
\hline 44 & & & $\overline{0 x a 9}$ & 129 & & & & & & & \\
\hline 45 & $x 3 f f f a c 2 c$ & & $0 \times a 7$ & 130 & & & $0 x f c$ & 215 & & & $0 \times 52$ \\
\hline 46 & $0 x 3 f f f a d 2 d$ & 12 & $0 \times a 5$ & 131 & $\overline{f f}$ & & $0 x f a$ & 216 & $d 7 d 7$ & 28 & $0 \times 50$ \\
\hline 47 & $0 x 3 f f f c$ & $d 1$ & $0 x a 3$ & 132 & $f f 8383$ & & $0 x f 8$ & 217 & $0 x 7 f f f d 8 d 8$ & 727 & $0 \times 4 e$ \\
\hline 48 & $0 \times 3$ & & & 133 & & & $\sqrt{0 x f 6}$ & 218 & & & $0 \times 4 c$ \\
\hline 49 & & & & 134 & & & & & & & \\
\hline 50 & $0 \times 3$ & & $0 x 9 d$ & 135 & & & $\sqrt{0 x f 2}$ & 220 & & & $0 \times 48$ \\
\hline 51 & $0 x 3$ & & & 136 & & & $f 0$ & 221 & & & \\
\hline 52 & & & $0 x$ & 137 & & & & & & & \\
\hline 53 & $0 x 3$ & & \begin{tabular}{|l|}
$0 \times 97$ \\
\end{tabular} & 138 & & & Oxec & 223 & & & $0 \times 42$ \\
\hline 54 & & & & 139 & & & & & & & \\
\hline 55 & & & & 140 & & & & 225 & & & \\
\hline 56 & $0 \times 3$ & & & 141 & & & & 226 & & & \\
\hline & & & & 142 & & & & & & & \\
\hline 58 & & & & 143 & & & $e 2$ & 228 & & & \\
\hline 59 & & & & 144 & & & & & & & \\
\hline 60 & 0 & & & 145 & & & & 23 & & & \\
\hline 61 & & & & 146 & & & & 231 & & & $0 x 32$ \\
\hline 62 & & & & 147 & & & & & & & \\
\hline 63 & & & \begin{tabular}{|l|}
$0 x 83$ \\
\end{tabular} & 148 & & & $0 x$ & 233 & & & $0 x 2 e$ \\
\hline 64 & & & & 149 & & & & 234 & & & \\
\hline 65 & & & & 15 & & & & & & & \\
\hline 66 & & & & 15 & & & & 236 & & & \\
\hline 67 & & & & 152 & & & & & & & \\
\hline 68 & & & $0 x$ & 153 & & & Oxce & 238 & ded & & $0 \times 24$ \\
\hline 69 & & & & 154 & & & $\sqrt{0 x c c}$ & 239 & & & $0 x 22$ \\
\hline 70 & & & & & & & & & & & \\
\hline 71 & & & & 1 & & & & & & & \\
\hline 72 & & & & 15 & & & & & & & \\
\hline 73 & $0 \times 3$ & & & 158 & $0 x$ & & $0 x{ }^{2}$ & 243 & & & \\
\hline 74 & $f f f c 949$ & & $0 \times 6 d$ & 159 & $0 x$ & & $0 x c 2$ & 244 & & & $0 \times 18$ \\
\hline 75 & & & & 160 & & & & 245 & & & \\
\hline 76 & $0 \times 3$ & & & 16 & 0 & & & & & & \\
\hline 77 & & & & 16 & & & & 24 & & & \\
\hline 78 & $0 x 3 f f f c d 4$ & & $0 x 65$ & 163 & $f f a 2 a 2$ & & $0 x b a$ & 248 & & $0 x 808$ & $0 \times 10$ \\
\hline 79 & $0 x 3 f f f c e 4 e$ & $b 1$ & $0 x 63$ & 164 & $0 x 7$ & & $0 \times b 8$ & 249 & & $0 \times 707$ & $0 x e$ \\
\hline 80 & & & & 165 & & & & 250 & & & \\
\hline 81 & & & & 16 & & & & 25 & & & \\
\hline 82 & & & & 167 & & & & 252 & & & \\
\hline 83 & $0 x 3 f f f f d$ & & $0 \times 5 b$ & 168 & ffa7a7 & & $0 \times 60$ & 253 & $0 \times 7$ & $0 \times 303$ & $0 \times 6$ \\
\hline 84 & $0 x 3 f f f d 353$ & $0 \times 40002 \mathrm{cac}$ & $0 \times 59$ & 169 & $0 x 7 f f f a 8 a 8$ & $0 \times 5757$ & $0 x a e$ & 254 & $0 x 7 f f f f d f d$ & $0 \times 202$ & $0 \times 4$ \\
\hline & & & & & & & & & & & \\
\hline
\end{tabular}




\section{B Generating Identical Keystreams for $\Delta i v_{1}$}

Here we describe more details of an algorithm that is used to generate identical keystreams for the IV difference at $i v_{1}$ :

1. Initialize $i v_{0}, i v_{1}, \ldots, i v_{15}$ with 0 . Set $i v_{13}=64$.

2. Denote $\left(i v_{4}+8 i v_{13}+16 i v_{10}\right)$ as sum $_{1}$ and guess sum $_{1}$ with 1 of the 6376 possible values.

3. Guess $i v_{2}[1,2]$, and compute $v$, until the condition $v[1 . .7]-(v>>8)[1 . .7] \leq 1$ is satisfied. If not possible, go to (2) .

4. Guess $i v_{7}$ and $i v_{11}$, and compute $u$, until $u[24 . .31]=0 \operatorname{xff}$ is satisfied. We store the intermediate state $s_{16}$. If not possible, go to (3).

5. Guess $i v_{15}$ and re-compute $u$, until $u[1 . .7]=u[9 . .15]$ and $u[8]=0$ are satisfied. If not possible, go to (4).

6. Now we compare the current $s_{16}$ with stored $s_{16}$ to capture the change. By properly changing $i v_{2}$ and $i v_{13}$ (this is the reason $i v_{13}$ is initialized as 64 ), we can always change the current $s_{16}$ back to the saved value. Hence, $u[24 . .31]$ will remain.

7. Determine $i v_{1}$ as follows:

- If $v[8] \neq v[16]$, then if $u[1 . .16]<v[1 . .16]$ is satisfied, $i v_{1}=256+u[1 . .16]-$ $v[1 . .16]$ and update $v$, otherwise, go to $(5)$.

- If $v[8]=v[16]$, then if $u[1 . .16]>=v[1 . .16]$ is satisfied, $i v_{1}=u[1 . .16]-$ $v[1 . .16]$ and update $v$, otherwise, go to (5).

8. Guess $i v_{0}, i v_{5}$ and $i v_{14}$, compute $v$, until $v[16 . .31]=0 x f f f f$. If not possible, go to $(5)$.

9. If $(u \oplus v)[1]=1$, let $i v_{2}=i v_{2} \oplus 2$. Choose $i v_{3}$ properly to ensure $u[16 . .23]=$ oxff. Check if we indeed have $v=u$, then output $i v_{0}, i v_{1}, \ldots, i v_{15}$. Otherwise, go to (8).

In this algorithm, we restrict the forms of $v$ and $u$ to those starting with $0 \times 7 f f f$ to reduce the search space. 